\title{
Efeito da irradiação e do armazenamento na disponibilidade de ferro em carne de cordeiro tratado com diferentes dietas
}

\author{
Effect of irradiation and storage in the iron availability in lamb meat treated with different diets
}

Adriana Régia Marques de SOUZA ${ }^{1}$, Solange Guidolin CANNIATTI-BRAZACA ${ }^{2 *}$, Valter ARTHUR ${ }^{1}$, Meylene Aparecida Luzia COUTO ${ }^{3}$

\begin{abstract}
Resumo
A irradiação é um método eficiente para aumentar a segurança microbiológica e preservar os nutrientes contidos nas carnes, entre eles o ferro. A forma de melhor absorção, ferro heme, deve ser preservada, havendo melhora na qualidade nutricional da carne armazenada. A dieta também pode influenciar nos teores e formas em que são encontrados os nutrientes na carne. O ferro é fornecido pela dieta, sendo essencial para os processos metabólicos como transporte de oxigênio, metabolismo oxidativo e crescimento celular. Amostras de carne de cordeiro tratado com diferentes dietas (controle, TAC1, TAC2 e sorgo), foram embaladas a vácuo, e irradiadas nas doses 0,2 e $4 \mathrm{kGy}$, usandose Irradiador Multipropósito com fonte de cobalto-60 $\left({ }^{60} \mathrm{Co}\right)$ e armazenadas a $4{ }^{\circ} \mathrm{C}$ por 15 dias. Os valores de ferro total e ferro heme foram medidos aos 0 e 15 dias de armazenamento. $\mathrm{O}$ armazenamento diminuiu os teores de ferro total (de 18,36 para $14,28 \mathrm{mg} .100 \mathrm{~g}^{-1}$ ) e de ferro heme (de 13,78 para 10,52 mg. $100 \mathrm{~g}^{-1}$ ). As dietas afetaram os níveis de ferro total e heme da carne, sendo que a dieta com sorgo foi a que apresentou maiores teores. A dose de $2 \mathrm{kGy}$ foi a dose que mais afetou os valores de ferro, independentemente dos tempos de armazenamento. Foi constatado que os teores de ferro total e heme variaram em função do tempo de armazenamento, das doses de irradiação e das dietas fornecidas aos cordeiros.
\end{abstract}

Palavras-chave: irradiação; ferro heme; ferro total; dietas; cordeiro.

\begin{abstract}
Irradiation is an efficient method to increase the microbiological safety and to maintain the nutrients such as iron in the meat. The best absorption form, heme iron, should be preserved in order to increase the nutritional quality of stored meat. The diet can alter the nutrients contents and form in the meat. The iron is provided from the diet and it is an essential element for the metabolic processes such as oxygen transport, oxidative metabolism, and cellular growth. Meat lamb samples treated with different diets (it controls, TAC1, TAC2 and sorghum) were wrapped to vacuous, and irradiated in the doses 0,2 and $4 \mathrm{kGy}$ and stored at $4{ }^{\circ} \mathrm{C}$ during 15 days. The values of total iron and heme

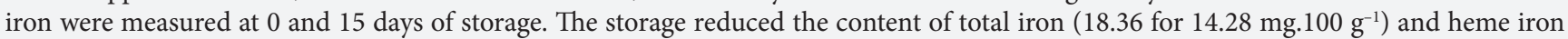
( 13.78 for $10.52 \mathrm{mg} .100 \mathrm{~g}^{-1}$ ). The diets affected the levels of total and heme iron of the meat, and the sorghum diet was the one that presented the larger content. The dose of $2 \mathrm{kGy}$ was the one that affected the iron the most independently of the storage time. It was verified that the amounts of total and heme iron varied according to the storage time, irradiation doses, and lamb diets.

Keywords: irradiation; heme iron; total iron; diets; lamb.
\end{abstract}

\section{Introdução}

Em regiões mais pobres do Brasil, os ovinos e os caprinos são fonte de proteína de origem animal, desempenhando função social expressiva. Por outro lado, embora a carne de ovinos não esteja entre as espécies de açougue mais consumidas no Brasil, a demanda desse produto diferenciado vem aumentando nos grandes centros urbanos da região Sudeste (PRADO, 1999). Se o animal é alimentado com uma determinada dieta, seus tecidos irão responder à composição dessa dieta ou à transformação metabólica que ocorre nos componentes dela (RUIZ et al., 2004). Aspectos que interferem na qualidade da carne e que estão associados aos fatores intrínsecos, peso ao abate e/ou idade, sexo e grupo genético, e dieta, podem ser manejados de forma a influenciar positivamente na qualidade da carne a ser ofertada ao mercado (SOUZA, 2001).

Uma alternativa para o controle de algumas verminoses em cordeiros é a utilização, na alimentação, de dietas ricas em taninos condensados. Taninos condensados são compostos fenólicos encontrados em várias forrageiras e árvores. Em baixas concentrações, alguns taninos condensados podem conferir vantagens nutricionais para ruminantes por redução de proteínas degradadoras no rúmen e aumento da circulação de

${ }^{1}$ Laboratório de Irradiação de Alimentos e Radioentomologia, Centro de Energia Nuclear na Agricultura - CENA, Universidade de São Paulo - USP, Av. Centenário, 303, CP 96, CEP 13416-000,Piracicaba-SP,Brasil,E-mail:acornel@cena.usp.br; arthur@cena.usp.br

2 Departamento de Agroindústria, Alimentos e Nutrição, Escola Superior de Agricultura Luiz de Queiroz - ESALQ, Universidade de São Paulo - USP, Av. Pádua Dias, 11, CP 9, CEP 13418-900, Piracicaba - SP, Brasil, E-mail: sgcbraza@esalq.usp.br

${ }^{3}$ Escola Superior de Agricultura Luiz de Queiroz - ESALQ, Universidade de São Paulo - USP, Av. Pádua Dias, 11, CP 9, CEP 13418-900, Piracicaba - SP, Brasil, E-mail: malcouto@esalq.usp.br

${ }^{*}$ A quem a correspondência deve ser enviada 
proteína e aminoácidos essenciais no intestino. O sorgo contém altos níveis de tanino e outros compostos fenólicos. Taninos e compostos fenólicos relacionados têm grande efeito antioxidante nos músculos do animal, melhorando a estabilidade oxidativa da carne (DU et al., 2002). Esses compostos seqüestram radicais livres, por isso são eficazes para prevenir a oxidação lipídica (SOARES, 2002). Porém, taninos e outros compostos fenólicos podem formar um complexo insolúvel com o ferro no trato intestinal e, com isso, reduzir a biodisponibilidade de ferro solúvel nas carnes dos animais (BRUNE; ROSSANDER; HALLBERG, 1989).

A irradiação é um método eficiente para aumentar a segurança para o consumidor, pois é eficaz contra patógenos e parasitas, preserva alimentos, melhora a qualidade pela diminuição da carga microbiana controlando as alterações causadas por elas, aumenta a vida útil dos produtos, propiciando a manutenção e a preservação dos nutrientes contidos nos alimentos. Porém pode influenciar significativamente de forma negativa na oxidação da carne e na coloração com o aumento da coloração vermelhoamarronzada. A coloração da carne fresca é determinada pelo estado do ferro heme. Algumas doses de irradiação, em geral doses altas, podem mudar o estado oxidativo do ferro heme e produzir ligantes que vão influenciar na qualidade da carne, levando à formação de uma coloração amarronzada (NAM; AHN, 2002).

O ferro, além de ser responsável pela coloração da carne, também é um elemento essencial para os processos metabólicos como transporte de oxigênio, metabolismo oxidativo e crescimento celular. O ferro está amplamente distribuído em alimentos. Alguns tecidos animais contêm ferro na forma heme, ferritina e hemosiderina (MARTINI, 2002). O ferro pode estar presente nos alimentos de duas formas: a forma heme que é encontrada em carnes e vísceras sob a forma de hemoglobina e mioglobina, e a forma não heme que é encontrada em alimentos de origem vegetal e animal que não seja hemoglobina e mioglobina (BIANCHI; SILVA; OLIVEIRA, 1992).

Dentre as formas de ferro heme e ferro não heme, sabe-se que parte do ferro presente em carnes (heme) é mais disponível biologicamente, podendo ser assimilado na proporção de aproximadamente $25 \%$ do total do ferro presente no alimento (COTRAN; KUMAN; ROBBINS, 1996).

O ferro não heme é considerado o mais importante promotor de oxidação de sistemas cárneos e sabe-se que proporciona formas químicas de ferro de grande importância. $\mathrm{O}$ aumento da quantidade de ferro não heme em carnes é considerado um reflexo da diminuição de ferro heme como conseqüência da quebra da molécula heme durante o cozimento ou estocagem (ESTÉVEZ; CAVA, 2004). O ferro heme é biologicamente mais disponível que o não heme, pois o não heme sofre influência de outras substâncias presentes na dieta, enquanto que a disponibilidade do ferro heme não é afetada pelas substâncias presentes. As principais fontes de ferro de origem animal são as carnes vermelhas e as vísceras, em especial o fígado (MARTÍNEZ et al., 1999).

O objetivo do estudo foi avaliar a disponibilidade do ferro total e do ferro heme nas carnes de cordeiros quando são submetidas a diferentes doses de radiação gama do ${ }^{60} \mathrm{Co}$, aos tempos de armazenamentos e às diferentes dietas fornecidas aos cordeiros.

\section{Material e métodos}

\subsection{Material}

Para o ensaio experimental, foram adquiridos 16 cordeiros da raça Santa Inês com dois meses de idade, mantidos a campo e divididos em quatro grupos de quatro animais, sendo um grupo controle (I), que recebeu somente feno; dois grupos (II e III), tratados com TAC (Taninos Altamente Condensados) na dosagem de 1,6 g. $\mathrm{kg}^{-1}$; e um grupo (IV), que recebeu sorgo. Os ovinos permaneceram alocados em quatro piquetes experimentais de mesmo tamanho, durante 60 dias. No final do dia e aos finais de semana, os cordeiros permaneceram estabulados e receberam concentrado (dieta total com $13 \%$ proteína bruta). Os cordeiros dos grupos II e III receberam dois dias consecutivos de TAC (primeiro e segundo dias experimentais); esse tratamento foi repetido após um mês.

O grupo I (controle) foi tratado com feno e concentrado (50-50\%). A suplementação da dieta para os grupos II e III foi feita com taninos altamente concentrados (TAC), extrato de Acácia molissima, sendo fornecido na dose de $1,6 \mathrm{~g} . \mathrm{kg}^{-1}$ de peso vivo. O grupo II (TAC1) foi suplementado com tanino por um dia, e o grupo III (TAC2), por dois dias. Os cordeiros foram abatidos com quatro meses e, após o abate, foi separada a paleta (Infraspinatus, Triceps brachii, Supraspinatus), a qual foi mantida sob refrigeração por 48 horas. As amostras foram desossadas, separadas em alíquotas (aproximadamente $50 \mathrm{~g}$ ), embaladas a vácuo em embalagens de polietileno de baixa densidade e, então, levadas para armazenamento a $4^{\circ} \mathrm{C}$ e irradiadas.

O irradiador utilizado no experimento foi o Multipropósito do IPEN (Instituto de Pesquisa Energéticas Nucleares), com fonte de cobalto-60 $\left({ }^{60} \mathrm{Co}\right)$ e a taxa de dose foi de $6 \mathrm{kGy} / \mathrm{h}$ (quiloGray/hora) (a taxa de dose foi medida através de dosímetro). As amostras receberam doses de 2 e $4 \mathrm{kGy}$ e foram analisadas no primeiro dia de irradiação (tempo 1) e após 15 dias (tempo 2). As amostras não irradiadas serviram de controle. Todas as amostras foram refrigeradas a $4{ }^{\circ} \mathrm{C}$.

\subsection{Análises de ferro}

\section{Determinação de ferro total}

A determinação do ferro total foi realizada utilizando-se o método Carpenter e Clark (1995), o qual se baseia na prédigestão das amostras em frasco do tipo Erlenmeyer com ácido nítrico concentrado por uma noite. Após a digestão, os frascos foram colocados sobre placa pré-aquecida até a evaporação do líquido. Após a evaporação, adicionaram-se alíquotas do reagente peróxido de hidrogênio e ácido sulfúrico, segundo Hatch et al. (1986), em cada amostra até que estas se tornaram claras, geralmente após 3 a 4 adições. Deixaram-se então os frascos sobre placa aquecida até que todo peróxido de hidrogênio fosse expelido e que vapores de ácido sulfúrico se tornassem evidentes. Após o resfriamento, transferiu-se o líquido dos Erlenmeyers 
para um balão volumétrico de $10 \mathrm{~mL}$, usando ácido clorídrico $0,01 \mathrm{~N}$ para enxágüe. As alíquotas foram analisadas pelo método da ferrozine, segundo Carter (1971) e Stookey (1970).

\section{Determinação do ferro heme}

A determinação do ferro heme foi realizada utilizando o método descrito por Hornsey (1956), com algumas modificações. O método baseia-se na extração por acetona acidificada. As amostras da carne de cordeiro $(5 \mathrm{~g})$ foram colocadas em tubos de centrífuga de $50 \mathrm{~mL}$ e adicionadas de $20 \mathrm{~mL}$ de acetona mais $0,5 \mathrm{~mL}$ de $\mathrm{HCl}$. A seguir, adicionou-se água deionizada até que o volume total de água na amostra fosse equivalente a $4,5 \mathrm{~g}$. Para a determinação da quantidade de água a ser adicionada, foi determinada a umidade do material segundo AOAC (2000). As amostras foram então centrifugadas (Centrífuga modelo TDL80-2B, marca Centribio ${ }^{\circ}$ ) a $2000 \mathrm{~g}$ por 15 minutos e filtradas em papel. A absorbância do filtrado foi medida a $640 \mathrm{~nm}$ e, em seguida, foi calculado o conteúdo de ferro heme.

\subsection{Análise estatística}

O delineamento estatístico empregado foi o inteiramente casualizado (3 doses de irradiação, 2 tempos, 4 dietas), com três repetições por tratamento. Os resultados foram submetidos à análise de variância, com teste de $F$. $O$ teste de Tukey foi realizado para as amostras que obtiveram significância no teste de $F$ em nível de $5 \%$, para que fossem avaliados os efeitos das diferentes doses nos tratamentos. Os cálculos estatísticos foram realizados pelo pacote estatístico SAS (1998).

\section{Resultados e discussão}

\subsection{Ferro total}

Os valores de ferro total encontrados na paleta, (Infraspinatus, Triceps brachii, Supraspinatus) de acordo com as diferentes dietas, doses de irradiação e tempo de armazenamento são mostrados nas Tabelas 1 e 2. Os resultados encontrados indicam decréscimo nos valores de ferro total aos 15 dias de armazenamento e que a dose de irradiação que mais afetou a quantidade de ferro foi a de $2 \mathrm{kGy}$, apresentando os maiores valores. Esse aumento foi devido à perda de água (Tabela 5) ou devido ao processo de exsudação que pode ter ocorrido na carne. De acordo com Carpenter e Clark (1995), os teores de ferro total e ferro heme da carne de cordeiros são de 16 e $14 \mu \mathrm{g} \cdot \mathrm{g}^{-1}$, respectivamente e, para Schricker, Miller e Stouffer (1982), os valores são de 16,4 e 9,4 $\mu \mathrm{g} . \mathrm{g}^{-1}$, para ferro total e ferro heme. Ambos os valores estão abaixo dos encontrados no presente trabalho, indicando que a alimentação dos animais influenciou consideravelmente nos nutrientes da carne.

Independentemente das dietas (Tabela 2), os valores de ferro total diminuíram com o aumento do tempo de estocagem e a dose de irradiação que apresentou maior efeito foi a dose $2 \mathrm{kGy}$, em ambos os tempos de armazenamento, elevando os valores de ferro.

Houve diminuição nos valores de ferro total para todas as dietas, independentemente das doses de irradiação, com o
Tabela 1. Efeito da irradiação e do armazenamento nos valores de ferro total (mg.100 g ${ }^{-1}$ da paleta (Infraspinatus, Triceps brachii, Supraspinatus) de cordeiros Santa Inês, tratados com diferentes dietas.

\begin{tabular}{cccc}
\hline Dieta & Doses (kGy) & Dia 0 & Dia 15 \\
\hline Controle & 0 & $14,42 \pm 0,6^{1 \mathrm{Baa}}$ & $14,28 \pm 0,4^{\mathrm{Ba}}$ \\
& 2 & $17,03 \pm 0,2^{\mathrm{Aa}}$ & $16,08 \pm 0,1^{\mathrm{Ab}}$ \\
& 4 & $14,95 \pm 0,6^{\mathrm{Ba}}$ & $14,58 \pm 0,5^{\mathrm{Ba}}$ \\
TAC1 $^{*}$ & 0 & $16,09 \pm 0,4^{\mathrm{Ca}}$ & $15,55 \pm 0,4^{\mathrm{Bb}}$ \\
& 2 & $17,21 \pm 0,3^{\mathrm{Ba}}$ & $16,19 \pm 0,4^{\mathrm{Ab}}$ \\
& 4 & $18,08 \pm 0,1^{\mathrm{Aa}}$ & $16,51 \pm 0,5^{\mathrm{Ab}}$ \\
TAC2 $^{*}$ & 0 & $17,65 \pm 0,4^{\mathrm{Ba}}$ & $15,39 \pm 0,2^{\mathrm{Bb}}$ \\
& 2 & $18,36 \pm 0,4^{\mathrm{Aa}}$ & $16,45 \pm 0,4^{\mathrm{Ab}}$ \\
& 4 & $17,71 \pm 0,1^{\mathrm{Ba}}$ & $15,55 \pm 0,2^{\mathrm{Bb}}$ \\
Sorgo & 0 & $17,74 \pm 0,3^{\mathrm{Ba}}$ & $15,68 \pm 0,2^{\mathrm{Bb}}$ \\
& 2 & $18,11 \pm 0,2^{\mathrm{Aa}}$ & $16,35 \pm 0,1^{\mathrm{Ab}}$ \\
& 4 & $16,09 \pm 0,2^{\mathrm{Ca}}$ & $15,21 \pm 0,2^{\mathrm{Bb}}$ \\
\hline
\end{tabular}

${ }^{1}$ Média de três repetições \pm desvio padrão; ${ }^{2}$ letras maiúsculas diferentes nas colunas indicam diferença significativa entre as doses dentro de cada dieta $(\mathrm{p} \leq 0,05)$; '3letras minúsculas diferentes nas linhas indicam diferenças significativas nos tempos de armazenamento $(\mathrm{p} \leq 0,05)$; $\mathrm{e}^{*}$ dietas suplementadas com taninos altamente concentrados, sendo fornecidas por um dia (TAC1) ou dois dias (TAC2).

Tabela 2. Efeito das diferentes dietas nos teores de ferro total (mg.100 g ${ }^{-1}$ ) da paleta (Infraspinatus, Triceps brachii, Supraspinatus) de cordeiros Santa Inês.

\begin{tabular}{cccc}
\hline Doses (kGy) & Dieta & Dia 0 & Dia 15 \\
\hline 0 & Controle $^{*}$ & $14,42 \pm 0,6^{1 \mathrm{~B} 2}$ & $14,28 \pm 0,4^{\mathrm{B}}$ \\
& TAC1 $^{*}$ & $16,09 \pm 0,4^{\mathrm{C}}$ & $15,55 \pm 0,4^{\mathrm{A}}$ \\
& TAC2 $^{*}$ & $17,65 \pm 0,4^{\mathrm{A}}$ & $15,39 \pm 0,2^{\mathrm{A}}$ \\
& Sorgo & $17,74 \pm 0,3^{\mathrm{A}}$ & $15,68 \pm 0,2^{\mathrm{A}}$ \\
2 & Controle $^{*}$ & $17,03 \pm 0,2^{\mathrm{B}}$ & $16,08 \pm 0,1^{\mathrm{B}}$ \\
& TAC1 $^{*}$ & $17,21 \pm 0,3^{\mathrm{B}}$ & $16,19 \pm 0,4^{\mathrm{B}}$ \\
& TAC2 $^{*}$ & $18,36 \pm 0,4^{\mathrm{A}}$ & $16,45 \pm 0,4^{\mathrm{A}}$ \\
& Sorgo $_{4}$ & $18,11 \pm 0,2^{\mathrm{A}}$ & $16,35 \pm 0,1^{\mathrm{A}}$ \\
& Controle $^{*}$ & $14,95 \pm 0,6^{\mathrm{D}}$ & $14,58 \pm 0,5^{\mathrm{C}}$ \\
& TAC1 $^{*}$ & $18,08 \pm 0,1^{\mathrm{A}}$ & $16,51 \pm 0,5^{\mathrm{A}}$ \\
& TAC2 $^{*}$ & $17,71 \pm 0,1^{\mathrm{B}}$ & $15,55 \pm 0,2^{\mathrm{B}}$ \\
& Sorgo $^{*}$ & $16,09 \pm 0,2^{\mathrm{C}}$ & $15,21 \pm 0,2^{\mathrm{B}}$ \\
\hline
\end{tabular}

${ }^{1}$ Média de três repetições \pm desvio padrão; ${ }^{2}$ letras maiúsculas diferentes nas colunas indicam diferença significativa entre as dietas dentro de cada dose ( $\mathrm{p} \leq 0,05)$; $\mathrm{e}^{*}$ dietas suplementadas com taninos altamente concentrados, sendo fornecidas por um dia (TAC1) ou dois dias (TAC2).

aumento do tempo de armazenamento da carne. O cozimento e o armazenamento alteram os teores de ferro heme, não heme e total em carnes, conforme comprova pesquisa realizada por Kongkachuichai, Napatthalung e Charoensiri (2002).

Antes do armazenamento, as amostras obtidas de animais que receberam as dietas TAC2 e sorgo foram semelhantes quanto ao teor de ferro total quando foram submetidas às doses de $0 \mathrm{e}$ $2 \mathrm{kGy}$, apresentando os maiores valores. As amostras obtidas das dietas TAC1 e TAC2 diferiram estatisticamente entre si, indicando que a quantidade de taninos fornecida aos animais teve influência significativa no teor de ferro total, pois os animais que receberam a dieta TAC2 consumiram o dobro de taninos que os da TAC1. 
A dieta que menos influenciou os valores de ferro total, apresentando os valores mais baixos, foi a dieta controle, indicando que não houve efeito inibitório dos taninos na biodisponibilidade de ferro da carne de cordeiros, quando esses foram tratados com as outras dietas estudadas, sendo, então, esse tipo de alimentação indicado para o tratamento de ovinos.

\subsection{Ferro heme}

Os valores de ferro heme encontrados na paleta (Infraspinatus, Triceps brachii, Supraspinatus), de acordo com as diferentes dietas, doses de irradiação e tempo de armazenamento (Tabela 3 e 4), indicam aumento nos valores de

Tabela 3. Valores de ferro heme (mg. $100 \mathrm{~g}^{-1}$ ) para paleta (Infraspinatus, Triceps brachii, Supraspinatus) de cordeiros Santa Inês, tratados com diferentes dietas, doses de irradiação e tempo de armazenagem.

\begin{tabular}{cccc}
\hline Dieta & Doses (kGy) & Dia 0 & Dia 15 \\
\hline Controle & 0 & $12,30 \pm 0,01^{1 \mathrm{~A} 2 a 3}$ & $10,53 \pm 0,05^{\mathrm{Ab}}$ \\
& 2 & $12,64 \pm 0,01^{\mathrm{Ba}}$ & $11,03 \pm 0,02^{\mathrm{Bb}}$ \\
& 4 & $12,56 \pm 0,02^{\mathrm{Ca}}$ & $10,78 \pm 0,04^{\mathrm{Cb}}$ \\
TAC1 $^{*}$ & 0 & $13,19 \pm 0,02^{\mathrm{Aa}}$ & $10,67 \pm 0,10^{\mathrm{Ab}}$ \\
& 2 & $13,59 \pm 0,01^{\mathrm{Ba}}$ & $11,27 \pm 0,21^{\mathrm{Bb}}$ \\
& 4 & $13,34 \pm 0,02^{\mathrm{Ca}}$ & $11,07 \pm 0,20^{\mathrm{Bb}}$ \\
TAC2 $^{*}$ & 0 & $12,89 \pm 0,01^{\mathrm{Aa}}$ & $10,52 \pm 0,06^{\mathrm{Ab}}$ \\
& 2 & $13,25 \pm 0,04^{\mathrm{Ba}}$ & $11,17 \pm 0,09^{\mathrm{Bb}}$ \\
& 4 & $13,03 \pm 0,01^{\mathrm{Ca}}$ & $10,85 \pm 0,01^{\mathrm{Cb}}$ \\
Sorgo & 0 & $13,78 \pm 0,01^{\mathrm{Aa}}$ & $11,17 \pm 0,25^{\mathrm{Ab}}$ \\
& 2 & $13,65 \pm 0,04^{\mathrm{Ba}}$ & $11,41 \pm 0,28^{\mathrm{Ab}}$ \\
& 4 & $13,62 \pm 0,07^{\mathrm{Ba}}$ & $11,20 \pm 0,32^{\mathrm{Ab}}$ \\
\hline
\end{tabular}

${ }^{1}$ Média de três repetições \pm desvio padrão; 'letras maiúsculas diferentes nas colunas indicam diferença significativa entre as doses dentro de cada dieta $(p \leq 0,05)$; ${ }^{3}$ letras minúsculas diferentes nas linhas indicam diferenças significativas nos tempos de armazenamento $(\mathrm{p} \leq 0,05)$; $\mathrm{e}^{*}$ dietas suplementadas com taninos altamente concentrados, sendo fornecidas por um dia (TAC1) ou dois dias (TAC2).

Tabela 4. Efeito das diferentes dietas nos teores de ferro heme da paleta (Infraspinatus, Triceps brachii, Supraspinatus) de cordeiros Santa Inês.

\begin{tabular}{|c|c|c|c|}
\hline Doses (kGy) & Dieta & Dia 0 & Dia 15 \\
\hline \multirow[t]{4}{*}{0} & Controle & $12,30 \pm 0,01^{1 \mathrm{~A} 2}$ & $10,53 \pm 0,05^{\mathrm{A}}$ \\
\hline & $\mathrm{TACl}^{*}$ & $13,19 \pm 0,02^{\mathrm{B}}$ & $10,67 \pm 0,10^{\mathrm{A}}$ \\
\hline & TAC2* & $12,89 \pm 0,01^{\mathrm{C}}$ & $10,52 \pm 0,06^{\mathrm{A}}$ \\
\hline & Sorgo & $13,78 \pm 0,01^{\mathrm{D}}$ & $11,17 \pm 0,25^{\mathrm{B}}$ \\
\hline \multirow[t]{4}{*}{2} & Controle & $12,64 \pm 0,01^{\mathrm{A}}$ & $11,02 \pm 0,02^{\mathrm{A}}$ \\
\hline & $\mathrm{TAC}^{*}$ & $13,59 \pm 0,01^{\mathrm{B}}$ & $11,27 \pm 0,21^{\mathrm{BC}}$ \\
\hline & TAC $2^{*}$ & $13,25 \pm 0,04^{\mathrm{C}}$ & $11,17 \pm 0,09^{\mathrm{AB}}$ \\
\hline & Sorgo & $13,65 \pm 0,04^{\mathrm{B}}$ & $11,41 \pm 0,28^{\mathrm{C}}$ \\
\hline \multirow[t]{4}{*}{4} & Controle & $12,56 \pm 0,02^{\mathrm{A}}$ & $10,78 \pm 0,04^{\mathrm{A}}$ \\
\hline & $\mathrm{TAC}^{*}$ & $13,34 \pm 0,02^{\mathrm{B}}$ & $11,08 \pm 0,20^{\mathrm{B}}$ \\
\hline & $\mathrm{TAC}^{*}$ & $13,03 \pm 0,01^{\mathrm{C}}$ & $10,85 \pm 0,01^{\mathrm{A}}$ \\
\hline & Sorgo & $13,62 \pm 0,07^{\mathrm{D}}$ & $11,20 \pm 0,32^{\mathrm{B}}$ \\
\hline
\end{tabular}

${ }^{1}$ Média de três repetições \pm desvio padrão; ${ }^{2}$ letras maiúsculas diferentes nas colunas indicam diferença significativa entre as dietas dentro de cada dose $(\mathrm{p} \leq 0,05)$; $\mathrm{e}^{*}$ dietas suplementadas com taninos altamente concentrados, sendo fornecidas por um dia (TAC1) ou dois dias (TAC2). ferro heme na dose de $2 \mathrm{kGy}$, independentemente do tempo de armazenamento. Quanto maior a perda de água no corte, menos concentrados ficam os teores de ferro, visto que pode haver perda do ferro junto com a água de exsudação (CHAIJAN et al., 2005). O aumento na perda de água foi observado antes da análise de ferro heme, quando a análise de umidade dos cortes foi realizada (Tabela 5). Segundo Shimokomaki et al. (2006), a mioglobina e outras proteínas heme são proteínas sarcoplasmáticas, estão localizadas dentro das células e são solúveis em água. Sendo assim, estas proteínas podem, em parte, ser perdidas com a ocorrência de exsudação, ou seja, durante a perda de umidade ou suco da carne. Quanto à influência do tempo, foi observado decréscimo nos valores de ferro aos 15 dias de armazenamento, indicando a degradação do ferro heme com a estocagem, o que está de acordo com Estévez e Cava (2004), que relatam que os valores de ferro heme diminuem com o passar do tempo.

Para todas as doses aplicadas, em ambos os tempos de armazenamento, a dieta que apresentou os maiores valores de ferro heme foi a dieta com sorgo. Isso indica que as dietas fornecidas aos animais estão fortemente ligadas à disponibilidade de ferro heme das carnes, podendo ser explicado pela formação de um complexo insolúvel com o ferro no trato intestinal que reduziu a biodisponibilidade de ferro solúvel, afetando dessa forma os teores de ferro heme (BRUNE; ROSSANDER; HALLBERG, 1989).

Segundo Estevez, Ventana e Cava (2006), compostos fenólicos são inibidores da degradação de proteínas. Esse fato não foi observado no presente trabalho, mas foi observada maior degradação das moléculas de heme nos tratamentos com dietas suplementadas com taninos condensados. Purchas et al. (2004), estudando o efeito da armazenagem refrigerada em carne bovina e de cordeiros, relataram que as causas da degradação de heme ainda não estão bem elucidadas, mas que há uma relação direta com as reações de oxidação.

Tabela 5. Valores de umidade das paletas irradiadas de cordeiros tratados com diferentes dietas.

\begin{tabular}{cccc}
\hline Dieta & Doses (kGy) & Dia 0 & Dia 15 \\
\hline Controle & 0 & $76,02 \pm 0,01^{1 \mathrm{Aza} 3}$ & $77,39 \pm 0,05^{\mathrm{Ab}}$ \\
& 2 & $74,28 \pm 0,01^{\mathrm{Ba}}$ & $73,71 \pm 0,02^{\mathrm{Bb}}$ \\
& 4 & $74,44 \pm 0,02^{\mathrm{Ca}}$ & $74,28 \pm 0,04^{\mathrm{Cb}}$ \\
\multirow{2}{*}{ TAC $^{*}$} & 0 & $76,14 \pm 0,02^{\mathrm{Aa}}$ & $76,24 \pm 0,10^{\mathrm{Ab}}$ \\
& 2 & $69,19 \pm 0,01^{\mathrm{Ba}}$ & $70,65 \pm 0,06^{\mathrm{Bb}}$ \\
& 4 & $71,41 \pm 0,02^{\mathrm{Ca}}$ & $72,51 \pm 0,03^{\mathrm{Bb}}$ \\
TAC $^{*}$ & 0 & $72,56 \pm 0,01^{\mathrm{Aa}}$ & $73,51 \pm 0,06^{\mathrm{Ab}}$ \\
& 2 & $60,36 \pm 0,04^{\mathrm{Ba}}$ & $63,93 \pm 0,09^{\mathrm{Bb}}$ \\
& 4 & $71,15 \pm 0,01^{\mathrm{Ca}}$ & $71,61 \pm 0,01^{\mathrm{Cb}}$ \\
Sorgo & 0 & $74,12 \pm 0,01^{\mathrm{Aa}}$ & $74,92 \pm 0,06^{\mathrm{Ab}}$ \\
& 2 & $72,59 \pm 0,02^{\mathrm{Ba}}$ & $73,68 \pm 0,08^{\mathrm{Ab}}$ \\
& 4 & $73,46 \pm 0,01^{\mathrm{Ba}}$ & $74,61 \pm 0,08^{\mathrm{Ab}}$
\end{tabular}

${ }^{1}$ Média de três repetições \pm desvio padrão; ${ }^{2}$ letras maiúsculas diferentes nas colunas indicam diferença significativa entre as doses dentro de cada dieta ( $\mathrm{p} \leq 0,05)$; ${ }^{3}$ letras minúsculas diferentes nas linhas indicam diferenças significativas nos tempos de armazenamento $(\mathrm{p} \leq 0,05)$; $\mathrm{e}^{*}$ dietas suplementadas com taninos altamente concentrados, sendo fornecidas por um dia (TAC1) ou dois dias (TAC2). 


\section{Conclusões}

No presente estudo, as dietas com diferentes quantidades de taninos afetaram as quantidades de ferro total e heme da carne, porém a dieta que se mostrou mais influente foi a dieta com suplementação de sorgo. $\mathrm{O}$ armazenamento refrigerado e a estocagem também afetaram as quantidades de ferro total e heme, diminuindo esses teores e afetando a disponibilidade do ferro, pois o ferro heme é absorvido sem interferência dos componentes da dieta, enquanto que o não heme tem interferência deles. A dose de irradiação de $2 \mathrm{kGy}$, foi a que apresentou maior influência nos valores de ferro da paleta de cordeiro Santa Inês, independentemente das dietas.

\section{Referências bibliográficas}

AOAC - Association of Official Analytical Chemists. Official Methods of Analysis of AOAC International. 17 ed. Method 950.46 Moisture in meat, 2000. Gaithersburg, 2000.

BIANCHI, M. L. P.; SILVA, H. C.; OLIVEIRA, J. E. D. Considerações sobre a biodisponibilidade de ferro dos alimentos. Archivos Latinoamericanos de Nutricion, v. 42, n. 2, p. 94-100, 1992.

BRUNE, M.; ROSSANDER, L.; HALLBERG, L. Iron absorption and phenolic compounds: importance of different phenolic structures. Journal of Clinical Nutrition, v. 43, n. 8, p. 547-558, 1989.

CARPENTER, C. E.; CLARK, E. Evaluation of methods used in meat iron analysis and iron content of raw and cooked meats. Journal of Agricultural and Food Chemistry, v. 43, n. 7, p. 1824-1827, 1995.

CARTER, P. Spectrophotometric determination of serum iron at the submicrogram level with a new reagent (Ferrozine). Analytical Biochemistry, v. 40, n. 2, p. 450-458, 1971.

CHAIJAN, M. et al. Changes of pigments and color in sardine (Sardinella gibbosa) and mackerel (Rastrelliger kanagurta) muscle during iced storage. Food Chemistry, v. 93, n. 4, p. 607-617, 2005.

COTRAN, R. S.; KUMAR, V.; ROBBINS, S. L. Patologia estrutural e functional. 5.ed. Rio de Janeiro: Guanabara, 1996.

DU, M.; CHERIAN, G.; STITT, P. A.; AHN, D. U. Effect of dietary sorghum cultivars on the storage stability of broiler breast and thigh meat. Poultry Science, v. 81, n. 9, p. 1385-1391, 2002.

ESTÉVEZ, M.; CAVA, R. Lipid and protein oxidation, release of iron from heme molecule and colour deterioration during refrigerated storage of liver pâté. Meat Science, v. 68, n. 4, p. 551-558, 2004.

ESTÉVEZ, M.; VENTANA, S.; CAVA, R. Effect of natural and synthetic antioxidants on protein oxidation and colour and texture changes in refrigerated stored porcine liver pâté. Meat Science, v. 74, n. 2, p. 396-403, 2006.

HATCH, G. E. et al. Nitrogen dioxide exposure and lung antioxidants in ascorbic acid-deficient Guinea Pigs. Toxicology and Applied Pharmacology, v. 82, n. 2, p. 351-359, 1986.

HORNSEY, H. C. The color of cooked cured pork. Journal of the Science of Food and Agriculture, v. 7, n. 8, p. 534-540, 1956.

KONGKACHUICHAI, R.; NAPATTHALUNG, P.; CHAROENSIRI, R. Heme and nonheme iron content of animal products commonly consumed in Thailand. Journal of Food Composition and Analysis, v. 15, n. 4, p. 389-398, 2002.

MARTÍNEZ, C. et al. Biodisponibilidad del hierro de los alimentos. Archivos Latinoamericanos de Nutricion, v. 49, n. 2, p. 106-113, 1999.

MARTINI, F. C. C. Comparação entre a disponibilidade de ferro na presença de vitamina $A$ e beta-caroteno em alimentos e medicamentos. Piracicaba, 2002. 113p. Dissertação - (Mestrado em Ciência dos Alimentos), Escola Superior de Agricultura "Luiz de Queiroz", Universidade de São Paulo.

NAM, K. C.; AHN, D. U. Carbon monoxide-heme pigment is responsible for the pink color in irradiated raw turkey breast meat. Meat Science, v. 60, n. 1, p. 25-23, 2002.

PRADO, O. V. Qualidade da carne de cordeiros Santa Inês e Bergamácia abatidos com diferentes pesos. Lavras, 1999. 109 p. Dissertação - (Mestrado em Zootecnia), Universidade Federal de Lavras - UFLA.

PURCHAS, R. W. et al. Cooking temperature effects on the forms of iron and levels of several other compounds in beef semitendinosus muscle. Meat Science, v. 68, n. 2, p. 201-207, 2004.

RUIZ, M. R. et al. Ácidos graxos essenciais (precursores) em carnes. Revista Nacional da Carne, v. 28, n. 332, p. 49-50, 2004.

SAS Institute. SAS user's guide: statistic. Version 6. 12 ed. Cary: SAS Institute, 1998. $846 \mathrm{p}$.

SCHRICKER, B. R.; MILLER, D. D; STOUFFER, J. R. Measurement and content of noheme and total iron in muscle. Journal of Food Science, v. 47, n. 7, p. 740-743, 1982.

SHIMOKOMAKI, M. et al. Atualidades em Ciência e Tecnologia de Carnes. 1 ed. São Paulo: Editora Varela, 2006.

SOARES, S. E. Ácidos fenólicos como antioxidantes. Revista de Nutrição, v. 15, n. 1, p. 71-81, 2002.

SOUZA, X. R. Efeitos de grupo genético, sexo e peso ao bate na qualidade de carne de cordeiros em crescimento. Lavras, 2001. 119p. Dissertação - (Mestrado em Ciência dos Alimentos), Universidade Federal de Lavras.

STOOKEY, L. L. A new spectrophotometric reagent for iron. Analytical Chemistry, v. 42, n. 7, p. 779-781, 1970. 